American Journal of Applied Sciences 9 (1): 54-59, 2012

ISSN 1546-9239

(C) 2012 Science Publications

\title{
An Overview of Research Issues in the Modern Healthcare Monitoring System Design using Wireless Body area Network
}

\author{
${ }^{1}$ D. Suresh and ${ }^{2} \mathrm{P}$. Alli \\ ${ }^{1}$ Department of CSE, PSNA College of Engg and Tech, Dindigul, India \\ ${ }^{2}$ Department of CSE, Velammal College of Engineering, Madurai, India
}

\begin{abstract}
Problem statement: Healthcare is recognized various leading edge technologies and new scientific discoveries to enable better cures for diseases and better means to enable early detection of most life threatening diseases. The modern health care focused for optimally reducing the healthcare costs. Approach: The modern healthcare system enables medical professionals to remotely perform real-time monitoring, early diagnosis and treatment for potential risky disease. A mobile patient monitoring system, which integrates current Personal Digital Assistant (PDA) technology and Wireless Local Area Network (WLAN) technology are proposed in the recent year. In addition to this, the medical diagnosis and patient consultations can be delivered via wire/wireless communication networks. Results: This study reviews on the recent research in the field of wireless body area networks and modern health-care systems. Conclusion: This study analyses the design, architecture and implementation of Wireless Body Area Network (WBAN) based health care system.
\end{abstract}

Key words: Health care industry, wireless body area network, wireless local area network, personal digital assistant, wireless sensor, Health Insurance Portability and Accountability Act (HIPAA), Wireless Body Area Network (WBAN), health care system

\section{INTRODUCTION}

Due to extreme growth of Healthcare Industry, U.S. Congress established Health Insurance Portability and Accountability Act (HIPAA) to protect workers and their families which includes major healthcare insurance for all illness and they provided the health insurance coverage also for change or lose of jobs. The hospitals, physicians, pharmaceutical companies and insurance providers that turned the market up to the \$2.3 trillion in the US healthcare system and these industries will be spending $\$ 55$ billion on telecommunications services over the next five years, says a new market research study released by the Insight study, expenditure by the US healthcare industry on telecommunications services will grow at a compounded rate of $8.4 \%$ over the forecast period, increasing from $\$ 7.5$ billion in 2008 to $\$ 11.3$ billion in 2013 .

The necessity of health care is increasing in every day for every person, regardless of age, class and background. The Table 1 shows the Thomson Reuter (2010) report, which clearly indicates the necessity of healthcare for variety of persons. The caring and disordered person and physically challenged persons are most important healthcare systems. The modern healthcare Research Corporation. According to the market analysis continuous monitoring of elderly persons, mentally

is applied for providing more efficient utilization of physicians, reducing the cost for hospital stays, reducing the skill level and minimizing the frequency of visits of home-care professionals, reducing hospital readmission rates and promoting health education at various levels. In addition to the above, the major requirement of the health care system is reducing risk.

Therefore, the modern healthcare system can provide a cheaper and smarter way to manage and care for patients suffering from age-related chronic diseases, such as heart disease, because chronic diseases require continuous, longterm monitoring rather than episodic assessments. In many of medical studies (Wisnicki, 2002; McHugh, 2004; Gururajan et al., 2004; Steele et al., 2009), they suggest that a continuous health monitoring system should be wearable and easy to use and they proposed a wearable, plug-and-play system using Bluetooth as the wireless communication protocol.

\begin{tabular}{|c|c|c|c|c|c|}
\hline \multicolumn{2}{|c|}{ Based on age } & \multicolumn{2}{|c|}{ Based on income } & \multicolumn{2}{|l|}{ Based on education } \\
\hline Age & $\begin{array}{l}(\%) \text { of } \\
\text { patients }\end{array}$ & Income & $\begin{array}{l}(\%) \text { of } \\
\text { patients }\end{array}$ & Education & $\begin{array}{l}(\%) \text { of } \\
\text { patients }\end{array}$ \\
\hline$<35$ & 32.20 & $<\$ 25 \mathrm{k}$ & 53.30 & High school or less & 65.10 \\
\hline $35-64$ & 21.20 & $\$ 25 \mathrm{k}-\$ 49.9 \mathrm{k}$ & 18.90 & Some college & 13.80 \\
\hline $65+$ & 30.20 & $\$ 50 \mathrm{k}-\$ 99.9 \mathrm{k}$ & 10.80 & College+ & 18.30 \\
\hline Total & 26.20 & Total & 26.20 & Total & 26.20 \\
\hline
\end{tabular}

Corresponding Author: D. Suresh, Department of CSE, PSNA College of Engg and Tech, Dindigul, India, 9965611326 
Am. J. Applied Sci., 9 (1): 54-59, 2012

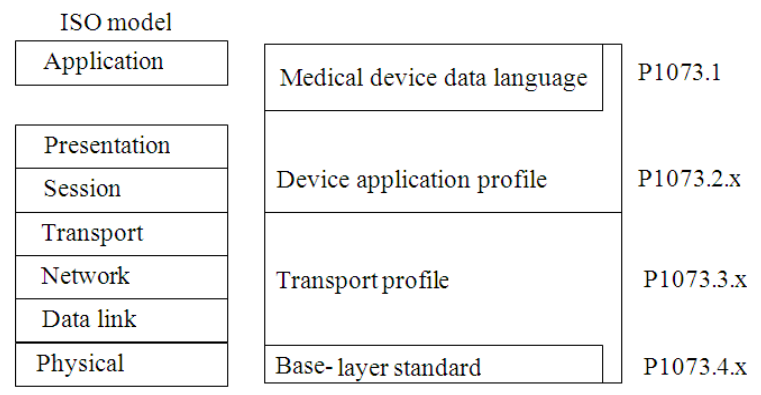

Fig. 1: IEEE standard for medi care applications

IEEE specifies IEEE $1073^{\mathrm{TM}}$ series for Medical Device Communications. IEEE 1073.3.1-Standard for Medical Device Communications, Transport ProfileConnection Mode. It defines the services and requirements for a bedside sub-network (Lo and Yang, 2005; Saeed et al., 2009). It defines services similar to those defined in Ethernet and TCP/IP. IEEE 1073.4.1Standard for Medical Device Communications, Physical Layer and Cable Connected. It defines the cables, connectors, data rates and bit level encoding for the MIB. It is coupled with IEEE 1073.3.1 to complete the "lower layers" for 1073. IEEE 1073.1-Standard for Medical Device Communications, Medical Device Data Language (MDDL) Overview and Framework. It defines the ISO Standards and conventions for using Object-Oriented Technology to define communications services for bedside medical devices. IEEE 1073.1.1 -Standard for Medical Device Communications, MDDL Common Definitions. It defines the common definitions for the object oriented communications services for medical devices.

IEEE 1073.1.2 -Standard for Medical Device Communications, MDDL Virtual Medical Device. It defines the general features of all virtual medical devices, in an object oriented environment. IEEE 1073.1.3 -Standard for Medical Device Communications, MDDL Virtual Medical Device. It defines the general features of specialized virtual medical devices for specific device categories. IEEE 1073.2 -Standard for Medical Device Communications, Application Profile, Overview and Framework. It describes the common theory and rules for all application profiles to be defined for the 1073 family. This was approved by the ballot group in late 1995 . ISO/IEEE 11073 -Point of Care Medical Device Communication Standards is a family of ISO, IEEE and CEN joint standards addressing the interoperability of medical devices. The Fig. 1 shows the layered approach of IEEE standard for Medicare applications.

\section{MATERIALS AND METHODS}

Telemedicine, a part of healthcare, is an information technology that enables doctors to perform medical consultations, diagnoses and treatments, as well as medical education, away from the patients. For example, doctors can remotely examine patients via remote viewing monitors and sound devices and/or sampling physiological data using telecommunication. Telemedicine technology is applied to emergency healthcare, video-consulting, telecardiology, telepath logy, teledermatology, teleophthalmo logy, teleoncology, telepsychiatry and teledentistry and continuous.

To address all telemedicine problem, apart from medication, higher and better usability with all communication devices such wired, wireless and sensory devices are mandatory (Steele et al., 2009). In order to coordinate with these varieties of devices, proposed new network architecture and a protocol to implement in the mesh routers, internet middle-boxes. This chapter describes briefly on various types of devices which are proposed for modern healthcare monitoring system.

The Fig. 2 shows the variety of sensor based wireless devices which are fixed in the various places of the human body for sensing and diagnosing. In general, the devices (McHugh, 2004) are classified as four types:

- Wrist watch type

- Chest belt type

- Shoulder type

- Necklace type

System design for WBAN: The biosensors and WBAN will be used in many applications including healthcare, sport and entertainment. From the wide spread application domains, healthcare applications require a group of biosensors which may be less in size. The data transmission medium of the WBAN system may be wired or wireless network. There are many challenges for researchers to implement the WBAN in robust, wearable, secure and scalable manner.

These research challenges include the size and power consumption of the biosensor, data rate, scalability in terms of the number of biosensors and also the number of patients.

The architectural block diagram of WBAN system is shown in Fig. 3. Several sensors are fixed on the body to collect data and pass it on via gateway, to the monitoring server. This monitoring server used for data to be stored, processed and analyzed.

The sensors in the architecture can be classified into two categories:

- Biosensors for the monitoring of vital physiological signs such as heart rate, oxygen level in the blood, blood pressure, rate of respiration and body temperature 


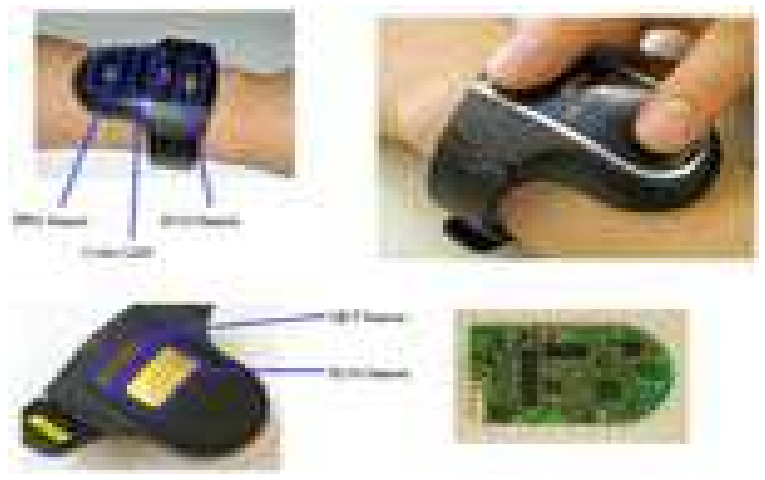

(a)

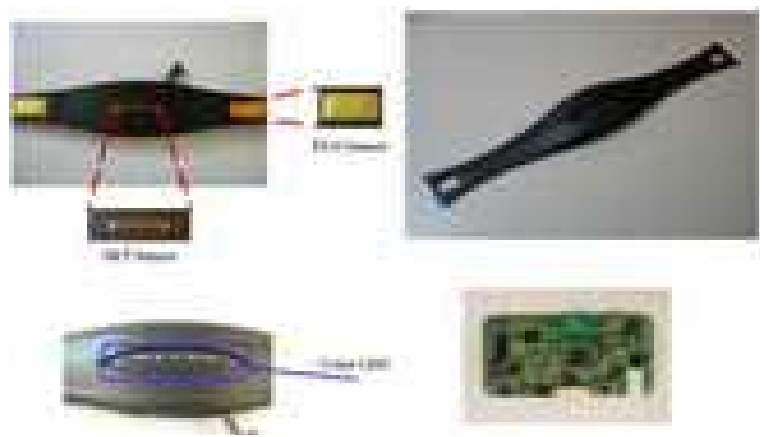

(b)

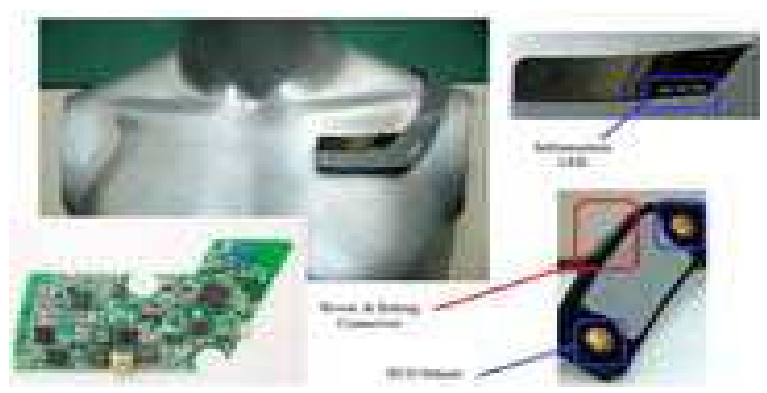

(c)

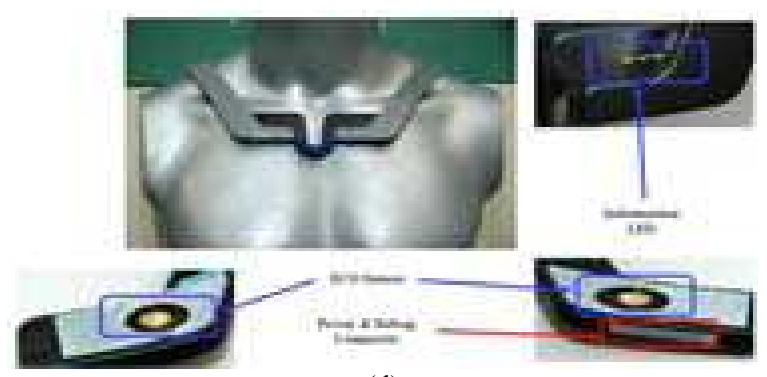

(d)

Fig. 2: Wearable physiological signal devices: (a) wrist watches type (b): chest belt type (c): shoulder type (d): necklace type

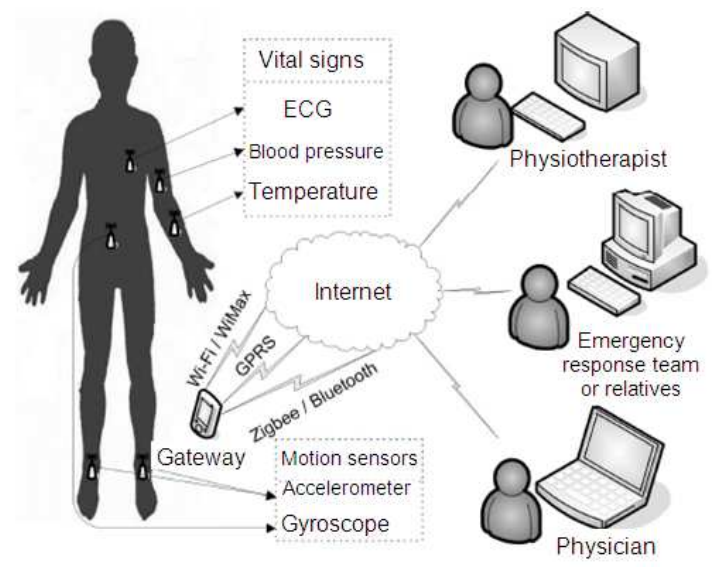

Fig. 3: Typical WBAN architecture

- Motion sensors for the collection of information about the current state of the patient's body such as walking, running, standing, sitting, falling. Brief explanations of these main units are follows

Biosensors: A wide range of biosensors can be found on the market, including sensors designed to monitor a person's heart rate or temperature, etc. and others that indicate whether a person is falling, bending and so forth. The hardware of these biosensors usually consists of a microcontroller, a few kilobytes of memory, an ultra-low power RF transceiver, antennae, sensors and actuators, analog signal conditioning circuitry, data converters and a battery module to power them. These biosensors need to run an operating system which, under the control of application logic, is responsible for (i) moving data between the data converter and the memory, (ii) formatting and encrypting data for transmission, (iii) and reliably transferring data through the RF transceiver. In addition, the OS is also responsible for task switching and managing the system's power.

Gateway: Biosensors communicate with the BAN controller, or gateway, which is the main interface between the body area network and the monitoring server. The gateway is responsible for collecting data from sensor nodes; storing data in the local memory in cases where there is no connection with the Internet; and forwarding data on its outgoing port to the Internet for eventual storage in the system's database. Due to the less stringent power requirements of the gateway (they can have large or rechargeable batteries); some of them have the ability to process different data streams and pass only relevant events to the system backend. The gateway is also responsible for the overall management of the BAN network, such as starting up a network with a unique network ID and allowing 
biosensors on the body to establish a connection with it and transfer data. The gateway can be a Personal Digital Assistant (PDA) with a Wi-Fi or Wi-MAX interface; a cell phone with a GPRS or UMTS interface; or a low-cost device with Zig-bee or Bluetooth interfaces for both collecting data from sensors and forwarding it to the Internet.

Monitoring server: A monitoring server consists of a database for data storage and processing and analyzing software for the delivery of the services for which the system is intended. Figure 3 shows a system where, for example, a physician, by examining the ECG signals of a patient, may suggest a further detailed diagnosis in hospital if need be; where a physiotherapist can monitor the rate of recovery of a patient who has fractured a limb in an accident; or where an emergency response team can provide immediate help in the event that an elderly person falls in her/his home. It is well understood that the bio metrics of each individual are very much unique. Thus, for effective processing a personalized profile should be "learned" automatically by the server. This is a crucial step towards minimizing the incidence of (and even achieving zerolevel of) false positives (i.e., raising the alarm in noncritical situations) and false negatives (i.e. missing a critical, perhaps life-threatening situation). To that end, a combination of innovative learning and reasoning algorithms are required to interpret data properly during monitoring.

\begin{tabular}{|c|c|c|c|}
\hline \multicolumn{2}{|c|}{ ControllerBits } & \multirow{2}{*}{$\frac{\text { Size }(\mathrm{L} \times \mathrm{W} \times \mathrm{H} \text { in } \mathrm{mm})}{35 \times 30 \times 12}$} & \multirow{2}{*}{$\begin{array}{l}\text { Wireless interface } \\
\text { Zigbee }\end{array}$} \\
\hline$\overline{A R M}$ & 32 & & \\
\hline AVR & 8 & $70 \times 55 \times 18$ & Bluetooth \\
\hline 8051 & 8 & $30 \times 25 \times 8$ & Custom \\
\hline MSP430 & 16 & $46 \times 3 \times 7$ & Zigbee \\
\hline PXA271 & 32 & $48 \times 36 \times 9$ & Zigbee \\
\hline
\end{tabular}

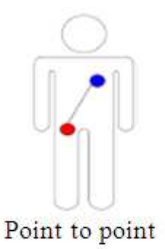

(a)

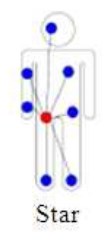

(b)

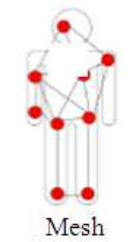

(c)

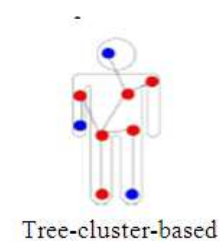

(d)

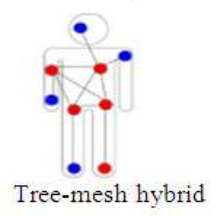

(e)
Fig. 4: Topology classification
Further to the basic studies on sensor nodes, the Table 2 compares various sensor nodes based on wireless interface and size.

Network topologies: The topologies available to be deployed for BANs are presented here. This study outlines their features and their suitability for possible applications. The following are various categories of sensor topologies:

- Point to point

- Star

- Mesh

- Tree (cluster-based)

- Tree-Mesh hybrid

Among the most common topologies for sensor networks are point-to-point, star, mesh, tree (clusterbased) and tree-mesh hybrid as depicted in Fig. 4? The simplest topology is point-to-point. It assumes all the devices have the same role(s) in the network. This topology is usually intended for a single link often between a data collector and a sensor sending its readings, such as a chest mounted sensor reporting to a hub device at the waist.

A star topology is one in which all the devices are directly connected through the central node. It has a potential to realize low latency and high bandwidth. However the hub is a single point of failure and the nodes can only communicate indirectly through the hub. Thus, a star topology has reduced reliability and limited scalability. A mesh topology comprises more capable and complex devices which have routing capability, so providing fault tolerance, self-healing and the ability to self-organize. It also facilitates scalability. Unfortunately it requires that all the devices are able to run advanced communication protocols/techniques, resulting in increased computation load and higher energy consumption.

A mesh topology would be suitable in all those scenarios where reliability and flexible communication are prioritized over energy efficiency and network longevity. A tree topology has a top "root" node with a branch/leaf structure below. A tree-mesh hybrid is a combination of tree and mesh topologies in which lower level nodes have dedicated connections up the tree to higher level nodes and full mesh connectivity onwards. Tree and tree-mesh hybrid topologies present an alternative to the (full) mesh topology, in all the cases where it is either infeasible for all the devices to be heavy-duty or the applications don't require such rich connectivity. The tree-related topologies exhibit good coverage, fault tolerance, low latency and a potential to realize high bandwidth. 


\section{RESULTS}

Warren et al. (2005); Fensli et al. (2005) proposes the interoperability of wireless body area network and the infrastructures of the health care systems. This study also provides the basic security model for the proposed healthcare system. Worldwide there are many research are carried out in the wireless body area network based healthcare systems, especially many organization like Wireless World Research Forum (WWRF) are initiated state of art architecture, design and implementation in this field of study. Warren et al. (2005) Motorola Emergency Medical Communications System, Smart Homes Foundation, New York City are few smart healthcare systems are implemented and tested in the industry. Mohan et al. (2008); Mohan and Baskaran (2010; 2011a; 2011b; 2011c; 2011d) proposed routing protocol using both artificial intelligence and swarm intelligence for wired cum wireless environment which offers optimality in all aspects.

\section{DISCUSSION}

The wireless sensor based modern healthcare system is very important for the advanced computer era. The sensors are highly advanced device which identifies, monitors and communicates even in a slight difference in the patient body condition. When applying sensor nodes, the person those are unable to communicate to the physician, such as mentally disordered persons and old aged persons, also easily monitored and forwarded the health condition by the automatic health-care monitoring system.

\section{CONCLUSION}

There are various sensors are available which are cost-effective, less in size and efficient for modern system. This study studies the various sensors and its specification to meet the requirement of modern healthcare system. And this study further reviews the recent research on wireless body area network based healthcare research. From the reviews, it is concluded that the sensor based healthcare is the future trend in the medical electronics field of study and more research is required to update the healthcare systems.

\section{REFERENCES}

Fensli, R., E. Gunnarson and T. Gundersen, 2005. A wearable ECG-recording system for continuous arrhythmia monitoring in a wireless tele-home-care situation. Proceedings of the 18th IEEE Symposium on Computer-Based Medical Systems, June, 23-24, IEEE Xplore Press, Norway, pp: 407412. DOI: $10.1109 / C B M S .2005 .22$

Gururajan, R., M. Toleman and J. Soar, 2004. Necessity for a new technology acceptance model to predict adoption of wireless technology in healthcare. University of Southern Queenslan.

Lo, B. and G.H. Yang, 2005. Architecture for body sensor networks. Perspective Pervasive Computing.

McHugh, J., 2004. A Chip in your Shoulde. Should I Get An RFID Implant? The Slate Group.

Mohan, B.C. and R. Baskaran, 2010, Improving network performance by optimal load balancing using ACO based redundant link avoidance algorithm. Int. J. Comput. Sci., 7: 27-35.

Mohan, B.C. and R. Baskaran, 2011a. Reliable transmission in network centric military networks. Eur. J. Sci. Res., 50: 564-574.

Mohan, B.C. and R. Baskaran, 2011b. Reliable BarrierFree Services (RBS) for heterogeneous next generation network. Adv. Power Electronics Instrumentat. Eng., 148: 79-82. DOI: 10.1007/9783-642-20499-9_13

Mohan, B.C. and R. Baskaran, 2011c. A survey: Ant colony optimization based recent research and implementation on several engineering domain. Expert Syst. Appl. DOI: 10.1016/j.eswa.2011.09.076

Mohan, B.C. and R. Baskaran, 2011d. Energy aware and energy efficient routing protocol for adhoc network using restructured artificial bee colony system, High Performance Architecture Grid Comput., 169: 473-484. DOI: 10.1007/978-3-64222577-2_65

Mohan, B.C., Sandeep, R. and D. Sridharan, 2008. A data mining approach for predicting reliable path for congestion free routing using self-motivated neural network. Software Eng. Artificial Intell. Network. Parallel/Distributed Comput., 149 : 237246. DOI: 10.1007/978-3-540-70560-4_20

Saeed, S., M. Faezipour, M. Nourani, S. Banerjee and G. Lee et al., 2009. A scalable wireless body area network for bio-telemetry. J. Inf. Proc. Syst., 5, pp.77-86. DOI: 10.3745/JIPS.2009.5.2.077

Steele, R., A. Lo, C. Secombe and Y.K. Wong, 2009. Elderly persons' perception and acceptance of using wireless sensor networks to assist healthcare. Int. J. Med. Infor., 7: 788-801. DOI: 10.1016/j.jijmedinf.2009.08.001 
Warren, S., J. Lebak, J. Yao, J. Creekmore and A. Milenkovic et al., 2005. Interoperability and security in wireless body area network infrastructures. Proceedings of the IEEE 27th Annual Conference on Engineering in Medicine and Biology Society, Jan. 17-18, IEEE Xplore Press, Shanghai, pp: 3837-3840. DOI: 10.1109/IEMBS.2005.1615297
Wisnicki, H.J., 2002. Wireless networking transforms health care: physicians' practices better able to handle work flow, increase productivity (The human connection). Highbeam Business. 